\title{
Nontrivial Solutions for a System of Second-Order Discrete Boundary Value Problems
}

\author{
Hua Su $\mathbb{D},{ }^{1}$ Yongqing Wang $\mathbb{D},^{2}$ and Jiafa $X u \mathbb{D}^{2}$ \\ ${ }^{1}$ School of Mathematics and Quantitative Economics, Shandong University of Finance and Economics, Jinan, Shandong 250014, \\ China \\ ${ }^{2}$ School of Mathematical Sciences, Qufu Normal University, Qufu, Shandong 273165, China
}

Correspondence should be addressed to Yongqing Wang; wyqing9801@163.com

Received 17 July 2020; Accepted 10 August 2020; Published 24 August 2020

Guest Editor: Chuanjun Chen

Copyright $(2020$ Hua Su et al. This is an open access article distributed under the Creative Commons Attribution License, which permits unrestricted use, distribution, and reproduction in any medium, provided the original work is properly cited.

In this work, we shall study the existence of nontrivial solutions for a system of second-order discrete boundary value problems. Under some conditions concerning the eigenvalues of relevant linear operator, we use the topological degree theory to obtain our main results.

\section{Introduction}

Nonlinear discrete problems appear in many mathematical models, such as computer science, mechanical engineering, control systems, economics, and fluid mechanics (see [1-4]). Owing to the wide applications, in recent years, there are a large number of researchers paying special attention in this direction (we refer to some results [5-15] and the references therein). For example, in [5], the authors used the Guo-Krasnosel'skii fixed point theorem to study the existence of positive solutions for the following second-order discrete boundary value problem:

$$
\left\{\begin{array}{l}
\Delta^{2} x_{i-1}+f\left(x_{i}\right)=0, \quad i \in[1, n] \\
x_{0}=0=x_{n+1}
\end{array}\right.
$$

and the following discrete second-order system:

$$
\left\{\begin{array}{l}
\Delta^{2} x_{i-1}+f\left(x_{i}, y_{i}\right)=0, \quad i \in[1, n], \\
\Delta^{2} y_{i-1}+g\left(x_{i}, y_{j}\right)=0, \quad i \in[1, n] \\
x_{0}=x_{n+1}=y_{0}=y_{n+1}=0
\end{array}\right.
$$

where $n$ is a positive integer, $[1, n]=\{1,2, \ldots, n\}, \Delta$ is the forward difference operator, i.e., $\Delta x_{i-1}=x_{i}-x_{i-1}$, and $\Delta^{2} x_{i-1}=\Delta\left(\Delta x_{i-1}\right)$.
In [6], the authors used the monotone iterative technique to investigate the existence and uniqueness of positive solutions for the following discrete $p$-Laplacian fractional boundary value problem:

$$
\left\{\begin{array}{l}
\Delta_{\nu-1}^{v}\left(\phi_{p}\left(\Delta_{\nu-1}^{\nu} y(t)\right)\right)=f(y(t+v-1)), \quad t \in[0, T]_{\mathbb{Z}}, \\
y(\nu-1)=y(\nu+T), \Delta_{\nu-1}^{v} y(\nu-1)=\Delta_{\nu-1}^{v} y(\nu+T)
\end{array}\right.
$$

where $v \in(0,1)$ is a real number, $\Delta_{\nu-1}^{v}$ is a discrete fractional operator, and $\phi_{p}(s)=|s|^{p^{-2}} s$ is the $p$-Laplacian with $s \in \mathbb{R}, p>1$.

Coupled systems of discrete problems have also been investigated by many authors; some results can be found in a series of papers [11-15] and the references cited therein (also see some results on differential systems [16-24]). For example, in [11], the authors used the Guo-Krasnosel'skii fixed point theorem to study the following systems of three-point discrete boundary value problems:

$\left\{\begin{array}{l}\Delta^{2} u(n-1)+\lambda a(n) f(u(n), v(n))=0, \quad n \in\{1,2, \ldots, N-1\} \\ \Delta^{2} v(n-1)+\mu b(n) g(u(n), v(n))=0 \\ u(0)=\beta u(\eta), u(N)=\alpha u(\eta), v(0)=\beta v(\eta), v(N)=\alpha v(\eta),\end{array}\right.$ 
where $N \geq 4, \eta \in\{1,2, \ldots, N-1\}, \alpha>0, \beta>0, \lambda, \mu>0$. They offered some values for the parameters $\lambda$, $\mu$ to yield a positive solution for the above system.

In [12], the authors used the fixed point index to study the positive solutions for the following system of first-order discrete fractional boundary value problems:

$$
\begin{cases}\Delta_{v-1}^{v} x(t)=f_{1}(t+v-1, x(t+v-1), y(t+v-1)), & t \in[0, T]_{\mathbb{Z}}, \\ \Delta_{v-1}^{v} y(t)=f_{2}(t+v-1, x(t+v-1), y(t+v-1)), & t \in[0, T]_{\mathbb{Z}}, \\ x(v-1)=x(v+T), y(v-1)=y(v+T) . & \end{cases}
$$

By discrete Jensen's inequality, the authors adopted some appropriate nonnegative concave and convex functions to characterize the coupling behavior of the nonlinearities $f_{i}(i=1,2)$.

Motivated by the aforementioned works, in this paper, by means of the topological degree theory, we study the existence of nontrivial solutions for the following system of second-order discrete boundary value problems:

$$
\left\{\begin{array}{l}
\Delta^{2} u(k-1)+f(k, v(k))=0, \quad k \in\{1,2, \ldots, T\} \\
\Delta^{2} v(k-1)+g(k, u(k))=0 \\
u(0)=u(T+1)=v(0)=v(T+1)=0
\end{array}\right.
$$

where $T>2$ is a fixed positive integer number, $\triangle u(k)=$ $u(k+1)-u(k), \Delta^{2} u(k)=\triangle(\Delta u(k))$, and $f, g:\{1,2, \ldots, T\} \times$ $\mathbb{R} \longrightarrow \mathbb{R}(\mathbb{R}:=(-\infty,+\infty))$ are continuous and satisfy the following conditions:

(H1) There exist three nonnegative functions $a_{i}(k), b_{i}(k)\left(b_{i}(k) \neq 0, k \in \mathbb{T}_{1}\right)$ and $\beta_{i}(i=1,2)$ on $\mathbb{R}^{+}$ such that

$$
\begin{aligned}
f(k, v) & \geq-a_{1}(k)-b_{1}(k) \beta_{1}(v), g(k, u) \\
& \geq-a_{2}(k)-b_{2}(k) \beta_{2}(u), \quad \forall u, v \in \mathbb{R}, t \in \mathbb{T}_{1},
\end{aligned}
$$

where $\mathbb{T}_{1}:=\{1,2, \ldots, T\}$.

(H2) $\lim _{|v| \longrightarrow+\infty} \beta_{1}(v) /|v|=0, \lim _{|u| \longrightarrow+\infty} \beta_{2}(u) /|u|=0$. (H3) $\liminf _{|v| \longrightarrow+\infty} f(k, v) /|v|>\lambda_{1}, \liminf _{|u| \longrightarrow+\infty} g(k$, $u) /|u|>\lambda_{1}$ uniformly on $k \in \mathbb{T}_{1}$, where $\lambda_{1}=4 \sin ^{2}$ $(\pi /(2 T+2))$.

(H4) $\lim \sup _{|v| \longrightarrow 0}|f(k, v)| /|v|<\lambda_{1}, \limsup _{|u| \longrightarrow 0} \mid g(k$, $u)|/| u \mid<\lambda_{1}$ uniformly on $k \in \mathbb{T}_{1}$.

Now, we state our main result here.

Theorem 1. Suppose that (H1)-(H4) hold. Then, (6) has at least one nontrivial solution.

\section{Preliminaries}

Let $E$ be the Banach space of real valued functions defined on the discrete interval $\mathbb{T}_{2}$ with the norm $\|u\|=\max _{k \in \mathbb{T}_{2}}|u(k)|$, where $\mathbb{T}_{2}:=\{0,1,2, \ldots, T+1\}$. Define the following sets:

$$
\begin{gathered}
P=\left\{u \in E: u(k) \geq 0, \quad \forall k \in \mathbb{T}_{2}\right\}, \\
P_{0}=\left\{u \in E: \min _{k \in \mathbb{T}_{1}} u(k) \geq \frac{1}{T}\|u\|\right\},
\end{gathered}
$$

and $B_{r}=\{x \in E:\|x\|<r\}$ for $r>0$. Then, $P, P_{0}$ are cones on $E$, and $B_{r}$ is an open ball in $E$.

Lemma 1 (see $[11,15])$. Let $h(k) \in C\left(\mathbb{T}_{1}\right)$. Then, the discrete boundary value problem

$$
\left\{\begin{array}{l}
\Delta^{2} u(k-1)+h(k)=0, \quad k \in \mathbb{T}_{1}, \\
u(0)=u(T+1)=0,
\end{array}\right.
$$

has a solution with the form

$$
u(k)=\sum_{l=1}^{T} G(k, l) h(l), \quad k \in \mathbb{T}_{2},
$$

where

$$
G(k, l)=\frac{1}{T+1} \begin{cases}l(T+1-k), & 1 \leq l \leq k-1 \leq T, \\ k(T+1-k), & 0 \leq k \leq l \leq T .\end{cases}
$$

Furthermore, $G(k, l)$ has the following properties (see $[13,15]):$

(i) $G(k, l)>0$ and $G(k, l)=G(l, k)$, for $(k, l) \in \mathbb{T}_{1} \times \mathbb{T}_{1}$.

(ii) $G(l, l) / T \leq G(k, l) \leq G(l, l)$, for $(k, l) \in \mathbb{T}_{1} \times \mathbb{T}_{1}$.

By Lemma 1, system (6) is equivalent to

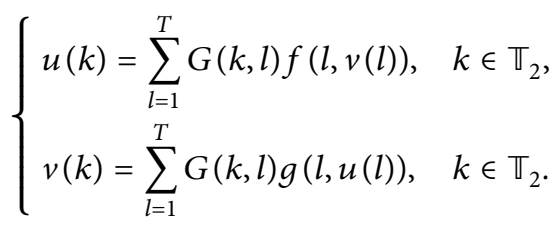

Then, we can define operators $\mathscr{T}, \mathcal{S}: E \longrightarrow E$ by

$$
\begin{aligned}
(\mathscr{T} v)(k) & =\sum_{l=1}^{T} G(k, l) f(l, v(l)),(\delta u)(k) \\
& =\sum_{l=1}^{T} G(k, l) g(l, u(l))
\end{aligned}
$$

and operator $\mathscr{A}: E \times E \longrightarrow E \times E$ by

$$
\mathscr{A}(u, v)(k)=((\mathscr{T} v),(\delta u))(k) .
$$

Note that $\mathscr{T}, \mathcal{S}, \mathscr{A}$ are completely continuous operators (see [11]), and $(u, v)$ solves (6) if and only if $(u, v)$ is a fixed point of the operator $\mathscr{A}$.

Lemma $2($ see $[7,15])$. Let $\phi(k)=\sin (k \pi) /(T+1), k \in \mathbb{T}_{2}$. Then, $\lambda_{1} \sum_{l=1}^{T} G(k, l) \phi(l)=\phi(k), \forall k \in \mathbb{T}_{1}$.

Define a linear operator as follows:

$$
(L x)(k)=\sum_{l=1}^{T} G(k, l) x(l), \quad \forall k \in \mathbb{T}_{2} .
$$


Then, we have

$$
(L \phi)(k)=\frac{1}{\lambda_{1}} \phi(k)
$$

and we have the following lemma.

Lemma 3. If $x \in P$, then $L x \in P_{0}$.

This is a direct result by Lemma 1 (ii), so we omit the proof.

Remark 1. $\phi \in P_{0}$ in Lemma 2.

Lemma 4 (see [25, Theorem A.3.3]). Let $\Omega$ be a bounded open set in a Banach space $E$ and $T: \Omega \longrightarrow E$ be a continuous compact operator. If there exists $x_{0} \in E \backslash\{0\}$ such that

$$
x-T x \neq \mu x_{0}, \quad \forall x \in \partial \Omega, \quad \mu \geq 0,
$$

then the topological degree $\operatorname{deg}(I-T, \Omega, 0)=0$.

Lemma 5 (see [25, Lemma 2.5.1]). Let $\Omega$ be a bounded open set in a Banach space $E$ with $0 \in \Omega$ and $T: \Omega \longrightarrow E$ be a continuous compact operator. If

$$
T x \neq \mu x, \quad \forall x \in \partial \Omega, \quad \mu \geq 1,
$$

then the topological degree $\operatorname{deg}(I-T, \Omega, 0)=1$.

\section{Main Results}

In order to obtain the Proof of Theorem 1, we first provide a lemma.

Lemma 6. There exists a sufficiently large $R>0$ such that

$$
\operatorname{deg}\left(I-\mathscr{A}, B_{R}, 0\right)=0 .
$$

Proof. By (H3), there exist $\varepsilon_{1}>0$ and $X_{1}>0$ such that

$$
\begin{array}{r}
f(k, v) \geq\left(\lambda_{1}+\varepsilon_{1}\right)|v|, g(k, u) \geq\left(\lambda_{1}+\varepsilon_{1}\right)|u|, \\
\forall k \in \mathbb{T}_{1},|u|,|v|>X_{1} .
\end{array}
$$

Note that when $k \in \mathbb{T}_{1},|u|,|v| \leq X_{1}$, the functions $|f(k, v)|$ and $|g(k, u)|$ are bounded, so we can choose some appropriate positive numbers $M_{1}, M_{2}$ such that

$$
f(k, v) \geq\left(\lambda_{1}+\varepsilon_{1}\right)|v|-M_{1}, g(k, u) \geq\left(\lambda_{1}+\varepsilon_{1}\right)|u|-M_{2},
$$

$$
\forall k \in \mathbb{T}_{1}, u, v \in \mathbb{R} \text {, }
$$

where

$$
\begin{aligned}
& M_{1}=\max _{k \in \mathbb{T}_{1},|u|,|v| \leq X_{1}}|f(k, v)|+\left(\lambda_{1}+\varepsilon_{1}\right) X_{1}, \\
& M_{2}=\max _{k \in \mathbb{T}_{1},|u|,|v| \leq X_{1}}|g(k, u)|+\left(\lambda_{1}+\varepsilon_{1}\right) X_{1} .
\end{aligned}
$$

From (H2), for any given $\varepsilon, \widetilde{\varepsilon}>0$ with $\varepsilon_{1}-\varepsilon\left\|b_{1}\right\|>0, \varepsilon_{1}-\widetilde{\varepsilon}\left\|b_{2}\right\|>0$, there is $X_{2}>X_{1}$ such that

$$
\beta_{1}(v) \leq \varepsilon|v|, \beta_{2}(u) \leq \widetilde{\varepsilon}|u|, \quad \forall|u|,|v|>X_{2} .
$$

Let $\beta_{1}^{*}=\max _{|v| \leq X_{2}} \beta_{1}(v)$ and $\beta_{2}^{*}=\max _{|u| \leq X_{2}} \beta_{2}(u)$. Then,

$$
\beta_{1}(v) \leq \varepsilon|v|+\beta_{1}^{*}, \beta_{2}(u) \leq \widetilde{\varepsilon}|u|+\beta_{2}^{*}, \quad u, v \in \mathbb{R} \text {. }
$$

Thus, we have

$$
\begin{array}{r}
f(k, v) \geq\left(\lambda_{1}+\varepsilon_{1}\right)|v|-a_{1}(k)-b_{1}(k) \beta_{1}(v)-M_{1} \\
\geq\left(\lambda_{1}+\varepsilon_{1}\right)|v|-a_{1}(k)-b_{1}(k)\left[\varepsilon|v|+\beta_{1}^{*}\right]-M_{1} \\
\geq\left(\lambda_{1}+\varepsilon_{1}-\varepsilon\left\|b_{1}\right\|\right)|v|-a_{1}(k)-\beta_{1}^{*} b_{1}(k)-M_{1}, \\
\forall k \in \mathbb{T}_{1}, v \in \mathbb{R},
\end{array}
$$

$$
\begin{array}{r}
g(k, u) \geq\left(\lambda_{1}+\varepsilon_{1}-\widetilde{\varepsilon}\left\|b_{2}\right\|\right)|u|-a_{2}(k)-\beta_{2}^{*} b_{2}(k)-M_{2}, \\
\forall k \in \mathbb{T}_{1}, u \in \mathbb{R} .
\end{array}
$$
let

Note that $\varepsilon, \widetilde{\varepsilon}$ can be chosen arbitrarily small, so we can

$$
R>\max \left\{N_{1}, N_{2}, N_{3}, N_{4}\right\},
$$

where

$$
\begin{aligned}
N_{1}= & \frac{2 \sum_{l=1}^{T} G(l, l)\left[a_{1}(l)+\beta_{1}^{*} b_{1}(l)+M_{1}\right]}{1-2 \varepsilon \sum_{l=1}^{T} G(l, l) b_{1}(l)}, \\
N_{2}= & \frac{2 \sum_{l=1}^{T} G(l, l)\left[a_{2}(l)+\beta_{2}^{*} b_{2}(l)+M_{2}\right]}{1-2 \widetilde{\varepsilon} \sum_{l=1}^{T} G(l, l) b_{2}(l)}, \\
N_{3}= & \frac{\left(\lambda_{1} T+(1+T)\left(\varepsilon_{1}-\varepsilon\left\|b_{1}\right\|\right)\right) \sum_{l=1}^{T} G(l, l)\left[a_{1}(l)+a_{2}(l)+\beta_{1}^{*} b_{1}(l)+\beta_{2}^{*} b_{2}(l)+M_{1}+M_{2}\right]}{\left(\varepsilon_{1}-\varepsilon\left\|b_{1}\right\|\right)-\left(\lambda_{1} T+(1+T)\left(\varepsilon_{1}-\varepsilon\left\|b_{1}\right\|\right)\right)\left[\varepsilon \sum_{l=1}^{T} G(l, l) b_{1}(l)+\widetilde{\varepsilon} \sum_{l=1}^{T} G(l, l) b_{2}(l)\right]}, \\
N_{4}= & \frac{\left(\lambda_{1} T+(1+T)\left(\varepsilon_{1}-\varepsilon\left\|b_{2}\right\|\right)\right) \sum_{l=1}^{T} G(l, l)\left[a_{1}(l)+a_{2}(l)+\beta_{1}^{*} b_{1}(l)+\beta_{2}^{*} b_{2}(l)+M_{1}+M_{2}\right]}{\left(\varepsilon_{1}-\varepsilon\left\|b_{2}\right\|\right)-\left(\lambda_{1} T+(1+T)\left(\varepsilon_{1}-\varepsilon\left\|b_{2}\right\|\right)\right)\left[\varepsilon \sum_{l=1}^{T} G(l, l) b_{1}(l)+\widetilde{\varepsilon} \sum_{l=1}^{T} G(l, l) b_{2}(l)\right]} .
\end{aligned}
$$


Now, we prove

$$
(u, v)-\mathscr{A}(u, v) \neq \mu(\phi, \phi), \quad \forall u, v \in \partial B_{R}, \mu \geq 0,
$$

where $\phi(k)=\sin (k \pi) /(T+1), k \in \mathbb{T}_{2}$. We argue this claim by indirection. Suppose that there exist $u, v \in \partial B_{R}, \mu \geq 0$ such that

$$
(u, v)-\mathscr{A}(u, v)=\mu(\phi, \phi)
$$

$u(k)=(\mathscr{T} v)(k)+\mu \phi(k)=\sum_{l=1}^{T} G(k, l) f(l, v(l))+\mu \phi(k)$

$$
v(k)=(\delta u)(k)+\mu \phi(k)=\sum_{l=1}^{T} G(k, l) g(l, u(l))+\mu \phi(k)
$$

$$
\begin{aligned}
& \widetilde{v}(k)=\sum_{l=1}^{T} G(k, l)\left[a_{1}(l)+b_{1}(l) \beta_{1}(v(l))+M_{1}\right], \\
& \tilde{u}(k)=\sum_{l=1}^{T} G(k, l)\left[a_{2}(l)+b_{2}(l) \beta_{2}(u(l))+M_{2}\right] .
\end{aligned}
$$

Then by Lemma 3, $\widetilde{u}, \widetilde{v} \in P_{0}$, and we also have

$$
\begin{aligned}
u(k)+\widetilde{v}(k)= & \sum_{l=1}^{T} G(k, l)\left[f(l, v(l))+a_{1}(l)\right. \\
& \left.+b_{1}(l) \beta_{1}(v(l))+M_{1}\right]+\mu \phi(k), \\
v(k)+\widetilde{u}(k)= & \sum_{l=1}^{T} G(k, l)\left[g(l, u(l))+a_{2}(l)\right. \\
& \left.+b_{2}(l) \beta_{2}(u(l))+M_{2}\right]+\mu \phi(k) .
\end{aligned}
$$

Using (24) and (25), we have

$$
\begin{aligned}
& f(l, v(l))+a_{1}(l)+b_{1}(l) \beta_{1}(v(l))+M_{1} \in P, \\
& g(l, u(l))+a_{2}(l)+b_{2}(l) \beta_{2}(u(l))+M_{2} \in P .
\end{aligned}
$$

So, from Lemma 3 and Remark 1, we have

$$
v+\widetilde{u}, u+\widetilde{v} \in P_{0} .
$$

Note that $u, v \in \partial B_{R}$, and using (24), $R>N_{1}$, and $R>N_{2}$, we have

$$
\begin{aligned}
\|\widetilde{v}\| & \leq \sum_{l=1}^{T} G(l, l)\left[a_{1}(l)+b_{1}(l) \beta_{1}(v(l))+M_{1}\right] \\
& \leq \sum_{l=1}^{T} G(l, l)\left[a_{1}(l)+b_{1}(l)\left(\varepsilon\|v\|+\beta_{1}^{*}\right)+M_{1}\right]<\frac{R}{2}, \\
\|\widetilde{u}\| & \leq \sum_{l=1}^{T} G(l, l)\left[a_{2}(l)+b_{2}(l)\left(\widetilde{\varepsilon}\|u\|+\beta_{2}^{*}\right)+M_{2}\right]<\frac{R}{2} .
\end{aligned}
$$

It is noted that $\|u\|=\|v\|=R, u+\widetilde{u}+\widetilde{v} \in P_{0}$, and $v+\widetilde{u}+\widetilde{v} \in P_{0}$. Therefore, we get

$$
\begin{aligned}
u(k)+\widetilde{u}(k)+\widetilde{v}(k) & \geq \frac{1}{T}\|u+\widetilde{u}+\widetilde{v}\| \geq \frac{1}{T}(\|u\|-\|\widetilde{u}+\widetilde{v}\|) \\
& \geq \frac{1}{T}[\|u\|-(\|\widetilde{u}\|+\|\widetilde{v}\|)], \\
v(k)+\widetilde{u}(k)+\widetilde{v}(k) & \geq \frac{1}{T}\|v+\widetilde{u}+\widetilde{v}\| \geq \frac{1}{T}(\|v\|-\|\widetilde{u}+\widetilde{v}\|) \\
& \geq \frac{1}{T}[\|v\|-(\|\widetilde{u}\|+\|\widetilde{v}\|)] .
\end{aligned}
$$

Using $R>N_{3}$, we have

$$
\begin{aligned}
& \left(\varepsilon_{1}-\varepsilon\left\|b_{1}\right\|\right) \sum_{l=1}^{T} G(k, l)[v(l)+\widetilde{u}(l)+\widetilde{v}(l)]-\left(\lambda_{1}+\varepsilon_{1}-\varepsilon\left\|b_{1}\right\|\right) \sum_{l=1}^{T} G(k, l)[\widetilde{u}(l)+\widetilde{v}(l)] \\
& \quad \geq \frac{\varepsilon_{1}-\varepsilon\left\|b_{1}\right\|}{T} \sum_{l=1}^{T} G(k, l)[R-(\|\widetilde{u}\|+\|\widetilde{v}\|)]-\left(\lambda_{1}+\varepsilon_{1}-\varepsilon\left\|b_{1}\right\|\right) \sum_{l=1}^{T} G(k, l)[(\|\widetilde{u}\|+\|\widetilde{v}\|)] \geq 0,
\end{aligned}
$$

and $R>N_{4}$ implies that

$$
\left(\varepsilon_{1}-\widetilde{\varepsilon}\left\|b_{2}\right\|\right) \sum_{l=1}^{T} G(k, l)[u(l)+\widetilde{u}(l)+\widetilde{v}(l)]-\left(\lambda_{1}+\varepsilon_{1}-\widetilde{\varepsilon}\left\|b_{2}\right\|\right) \sum_{l=1}^{T} G(k, l)[\widetilde{u}(l)+\widetilde{v}(l)] \geq 0 .
$$


Consequently, we obtain

$$
\begin{aligned}
& (\mathscr{T} v)(k)+\widetilde{v}(k)=\sum_{l=1}^{T} G(k, l)\left[f(l, v(l))+a_{1}(l)+b_{1}(l) \beta_{1}(v(l))+M_{1}\right] \\
& \geq \sum_{l=1}^{T} G(k, l)\left[\left(\lambda_{1}+\varepsilon_{1}-\varepsilon\left\|b_{1}\right\|\right)|v(l)|-a_{1}(l)-\beta_{1}^{*} b_{1}(l)-M_{1}+a_{1}(l)+b_{1}(l) \beta_{1}(v(l))+M_{1}\right] \\
& \geq \sum_{l=1}^{T} G(k, l)\left[\left(\lambda_{1}+\varepsilon_{1}-\varepsilon\left\|b_{1}\right\|\right)|v(l)|-\beta_{1}^{*} b_{1}(l)+b_{1}(l)\left(\varepsilon|v(l)|+\beta_{1}^{*}\right)\right] \geq\left(\lambda_{1}+\varepsilon_{1}-\varepsilon\left\|b_{1}\right\|\right) \sum_{l=1}^{T} G(k, l)|v(l)| \\
& \geq\left(\lambda_{1}+\varepsilon_{1}-\varepsilon\left\|b_{1}\right\|\right) \sum_{l=1}^{T} G(k, l)[v(l)+\widetilde{u}(l)+\widetilde{v}(l)]-\left(\lambda_{1}+\varepsilon_{1}-\varepsilon\left\|b_{1}\right\|\right) \sum_{l=1}^{T} G(k, l)[\widetilde{u}(l)+\widetilde{v}(l)] \\
& \geq \lambda_{1} \sum_{l=1}^{T} G(k, l)[v(l)+\widetilde{u}(l)+\widetilde{v}(l)] \geq \lambda_{1} \sum_{l=1}^{T} G(k, l)[v(l)+\widetilde{u}(l)] \\
& (\delta u)(k)+\tilde{u}(k)=\sum_{l=1}^{T} g(k, l)\left[g(l, v(l))+a_{2}(l)+b_{2}(l) \beta_{2}(u(l))+M_{2}\right] \\
& \geq \sum_{l=1}^{T} G(k, l)\left[\left(\lambda_{1}+\varepsilon_{1}-\widetilde{\varepsilon}\left\|b_{2}\right\|\right)|u(l)|-a_{2}(l)-\beta_{2}^{*} b_{2}(l)-M_{2}+a_{2}(l)+b_{2}(l) \beta_{2}(u(l))+M_{2}\right] \\
& \geq\left(\lambda_{1}+\varepsilon_{1}-\widetilde{\varepsilon}\left\|b_{2}\right\|\right) \sum_{l=1}^{T} G(k, l)|u(l)| \\
& \geq\left(\lambda_{1}+\varepsilon_{1}-\widetilde{\varepsilon}\left\|b_{2}\right\|\right) \sum_{l=1}^{T} G(k, l)[u(l)+\widetilde{u}(l)+\widetilde{v}(l)]-\left(\lambda_{1}+\varepsilon_{1}-\widetilde{\varepsilon}\left\|b_{2}\right\|\right) \\
& \cdot \sum_{l=1}^{T} G(k, l)[\widetilde{u}(l)+\widetilde{v}(l)] \geq \lambda_{1} \sum_{l=1}^{T} G(k, l)[u(l)+\widetilde{u}(l)+\widetilde{v}(l)] \\
& \geq \lambda_{1} \sum_{l=1}^{T} G(k, l)[u(l)+\widetilde{v}(l)] .
\end{aligned}
$$

As a result, we get

$(\mathscr{T} v)(k)+(\mathcal{S} u)(k)+\widetilde{u}(k)+\widetilde{v}(k) \geq \lambda_{1}(L(u+v+\widetilde{u}+\widetilde{v}))(k)$.

In view of (31) and (32), we see

$$
\begin{aligned}
u(k)+v(k)+\widetilde{u}(k)+\widetilde{v}(k)= & (\mathscr{T} v)(k)+(\delta u)(k)+\widetilde{u}(k) \\
& +\widetilde{v}(k)+2 \mu \phi(k) \\
\geq & \lambda_{1}(L(u+v+\widetilde{u}+\widetilde{v}))(k) \\
& +2 \mu \phi(k) \geq 2 \mu \phi(k) .
\end{aligned}
$$

Define $\quad \mu^{*}=\sup S_{\mu}:=\sup \{\mu>0: u+v+\widetilde{u}+\widetilde{v} \geq 2 \mu \phi\}$. Then, $S_{\mu} \neq \varnothing, \quad \mu^{*} \geq \mu$ and $u+v+\widetilde{u}+\widetilde{v} \geq 2 \mu^{*} \phi$. From $\phi=\lambda_{1} L \phi$, we obtain

$$
\lambda_{1} L(u+v+\widetilde{u}+\widetilde{v}) \geq \lambda_{1} L\left(2 \mu^{*} \phi\right)=2 \mu^{*} \lambda_{1} L \phi=2 \mu^{*} \phi .
$$

\section{Hence,}

$u+v+\widetilde{u}+\widetilde{v} \geq \lambda_{1} L(u+v+\widetilde{u}+\widetilde{v})+2 \mu \phi \geq 2\left(\mu^{*}+\mu\right) \phi$,

which contradicts the definition of $\mu^{*}$. Therefore, (29) holds, and from Lemma 4, we obtain

$$
\operatorname{deg}\left(I-\mathscr{A}, B_{R}, 0\right)=0 .
$$

This completes the proof.

Proof of Theorem 1. From (H4), there exist $\varepsilon_{2} \in\left(0, \lambda_{1}\right)$ and $r \in(0, R)$ such that

$$
\begin{array}{r}
|f(k, v)| \leq\left(\lambda_{1}-\varepsilon_{2}\right)|v|,|g(k, u)| \leq\left(\lambda_{1}-\varepsilon_{2}\right)|u|, \\
\forall k \in \mathbb{T}_{1}, u, v \in \mathbb{R} \text { with }|u|,|v| \leq r .
\end{array}
$$

This implies that 


$$
\begin{aligned}
& |(\mathscr{T} v)(k)|=\left|\sum_{l=1}^{T} G(k, l) f(l, v(l))\right| \leq \sum_{l=1}^{T} G(k, l)|f(l, v(l))| \leq\left(\lambda_{1}-\varepsilon_{2}\right) \sum_{l=1}^{T} G(k, l)|v(l)|, \\
& |(\mathcal{S u})(k)|=\left|\sum_{l=1}^{T} G(k, l) g(l, u(l))\right| \leq \sum_{l=1}^{T} G(k, l)|g(l, u(l))| \leq\left(\lambda_{1}-\varepsilon_{2}\right) \sum_{l=1}^{T} G(k, l)|u(l)| .
\end{aligned}
$$

Consequently, we have

$$
|(\mathscr{T} v)(k)|+|(\delta u)(k)| \leq\left(\lambda_{1}-\varepsilon_{2}\right) \sum_{l=1}^{T} G(k, l)[|u(l)|+|v(l)|] .
$$

Now, we prove that

$$
(u, v) \neq \mu \mathscr{A}(u, v)
$$

for all $u, v \in \partial B_{r}$ and $\mu \in[0,1]$. We argue by contradiction. Suppose that there exist $u, v \in \partial B_{r}$ and $\mu \in[0,1]$ such that

$$
(u, v)=\mu \mathscr{A}(u, v) \text {. }
$$

Therefore,

$$
u(k)=\mu(\mathscr{T} v)(k), \text { and } v(k)=\mu(\mathcal{S u})(k), \quad k \in \mathbb{T}_{1} .
$$

Hence, we have

$$
\begin{aligned}
|u(k)|+|v(k)| & \leq|(\mathscr{T} v)(k)|+|(\mathcal{S u})(k)| \\
& \leq\left(\lambda_{1}-\varepsilon_{2}\right) \sum_{l=1}^{T} G(k, l)[|u(l)|+|v(l)|] .
\end{aligned}
$$

From Lemma 1 (i) and Lemma 2, we have

$$
\lambda_{1} \sum_{k=1}^{T} G(k, l) \phi(k)=\phi(l), \quad \forall l \in \mathbb{T}_{1} .
$$

Multiplying both sides of (53) by $\sin (k \pi) /(T+1)$, then summing from 1 to $T$, and using (54), we obtain

$$
\sum_{k=1}^{T}[|u(k)|+|v(k)|] \frac{\sin (k \pi)}{(T+1)} \leq\left(\lambda_{1}-\varepsilon_{2}\right) \sum_{k=1}^{T} \frac{\sin (k \pi)}{(T+1)} \sum_{l=1}^{T} G(k, l)[|u(l)|+|v(l)|]=\frac{\lambda_{1}-\varepsilon_{2}}{\lambda_{1}} \sum_{l=1}^{T}[|u(l)|+|v(l)|] \frac{\sin (l \pi)}{(T+1)}
$$

This implies that

$$
\sum_{k=1}^{T}[|u(k)|+|v(k)|] \frac{\sin (k \pi)}{(T+1)}=0 .
$$

Because $\sin (k \pi) /(T+1) \geq 0(\not \equiv 0)$ for $k \in \mathbb{T}_{1}$, we have $|u(k)|+|v(k)| \equiv 0, k \in \mathbb{T}_{1}$. This contradicts $u, v \in \partial B_{r}$. Therefore, (50) holds, and Lemma 5 implies that

$$
\operatorname{deg}\left(I-\mathscr{A}, B_{r}, 0\right)=1 .
$$

Combining this with Lemma 6, we have

$$
\operatorname{deg}\left(I-\mathscr{A}, \frac{B_{R}}{\bar{B}_{r}}, 0\right)=\operatorname{deg}\left(I-\mathscr{A}, B_{R}, 0\right)-\operatorname{deg}\left(I-\mathscr{A}, B_{r}, 0\right)=-1 .
$$

Therefore, the operator $\mathscr{A}$ has at least one fixed point in $B_{R} / \bar{B}_{r}$, and (6) has at least one nontrivial solution. This completes the proof.

\section{Data Availability}

No data were used to support this study.

\section{Conflicts of Interest}

The authors declare that there are no conflicts of interest regarding the publication of this paper.

\section{Authors' Contributions}

This study was carried out in collaboration among all authors. All authors read and approved the final manuscript.

\section{Acknowledgments}

This study was supported by the project of National Social Science Fund of China (NSSFC) (18BTY015) and Shandong Province Higher Educational Science and Technology Program (J16LI01).

\section{References}

[1] R. P. Agarwal, Difference Equations and Inequalities, Theory, Methods, and Applications, Marcel Dekker Incorporated, New York, NY, USA, 2000.

[2] R. P. Agarwal and P. J. Y. Wong, Advanced Topics in Difference Equations, Kluwer Academic Publishers, Dordrecht, The Netherlands, 1997.

[3] S. Elaydi, An Introduction to Difference Equations, SpringerVerlag, New York, NY, USA, 3rd edition, 2011.

[4] W. G. Kelly and A. C. Peterson, Difference Equations: An Introduction with Applications, Academic Press, San Diego, CA, USA, 1991.

[5] G. Zhang and S. Ge, "Existence of positive solutions for a class of discrete Dirichlet boundary value problems," Applied Mathematics Letters, vol. 48, pp. 1-7, 2015.

[6] W. Cheng, J. Xu, D. O'Regan, and Y. Cui, "Positive solutions for a nonlinear discrete fractional boundary value problems 
with a $p$-Laplacian operator," Journal of Analysis Applications and Computational, vol. 9, no. 5, pp. 1959-1972, 2019.

[7] H. Lu, D. O'Regan, and R. Agarwal, "A positive solution for singular discrete boundary value problems with signchanging nonlinearities," Journal of Applied Mathematics and Stochastic Analysis, vol. 2006, pp. 1-14, 2006.

[8] K. Zhang, D. O’Regan, and Z. Fu, "Nontrivial solutions for boundary value problems of a fourth order difference equation with sign-changing nonlinearity," Advances in Difference Equations, vol. 2018, no. 1, p. 370, 2018.

[9] T. Sitthiwirattham, "Existence and uniqueness of solutions of sequential nonlinear fractional difference equations with three-point fractional sum boundary conditions," Mathematical Methods in the Applied Sciences, vol. 38, no. 13, pp. 2809-2815, 2005.

[10] C. S. Goodrich, "On semipositone discrete fractional boundary value problems with non-local boundary conditions," Journal of Difference Equations and Applications, vol. 19, no. 11, pp. 1758-1780, 2013.

[11] J. Henderson, S. K. Ntouyas, and I. K. Purnaras, "Positive solutions for systems of nonlinear discrete boundary value problems," Journal of Difference Equations and Applications, vol. 15 , no. 10 , pp. 895-912, 2009.

[12] J. Xu, C. S. Goodrich, and Y. Cui, "Positive solutions for a system of first-order discrete fractional boundary value problems with semipositone nonlinearities," Revista de la Real Academia de Ciencias Exactas, Físicas y Naturales. Serie A. Matemáticas, vol. 113, no. 2, pp. 1343-1358, 2019.

[13] J.-P. Sun and W.-T. Li, "Multiple positive solutions of a discrete difference system," Applied Mathematics and Computation, vol. 143, no. 2-3, pp. 213-221, 2003.

[14] W. Cheng, J. Xu, Y. Cui, and Q. Ge, "Positive solutions for a class of fractional difference systems with coupled boundary conditions," Advances in Difference Equations, vol. 2019, no. 1, p. 249, 2019.

[15] Y. Ding, J. Xu, and Z. Wei, "Positive solutions for a system of discrete boundary value problem," Bulletin of the Malaysian Mathematical Sciences Society, vol. 38, no. 3, pp. 1207-1221, 2015.

[16] Y. Li, J. Liu, D. O’Regan, and J. Xu, "Nontrivial solutions for a system of fractional q-difference equations involving q-integral boundary conditions," Mathematics, vol. 8, no. 5, p. 828, 2020.

[17] H. Zhang, Y. Li, and J. Xu, "Positive solutions for a system of fractional integral boundary value problems involving Hadamard-type fractional derivatives," Complexity, vol. 2019, p. 204, 2019.

[18] T. Qi, Y. Liu, and Y. Zou, "Existence result for a class of coupled fractional differential systems with integral boundary value conditions," The Journal of Nonlinear Sciences and Applications, vol. 10, no. 7, pp. 4034-4045, 2017.

[19] T. Qi, Y. Liu, and Y. Cui, "Existence of solutions for a class of coupled fractional differential systems with nonlocal boundary conditions," Journal of Function Spaces, vol. 2017, pp. 1-9, 2017.

[20] J. Jiang, D. O’Regan, J. Xu, and Z. Fu, "Positive solutions for a system of nonlinear Hadamard fractional differential equations involving coupled integral boundary conditions," Journal of Inequalities and Applications, vol. 2019, no. 1, p. 204, 2019.

[21] W. Yang, "Positive solutions for nonlinear semipositone fractional q-difference system with coupled integral boundary conditions," Applied Mathematics and Computation, vol. 244, pp. 702-725, 2014.
[22] X. Zhang, Y. Wu, and L. Caccetta, "Nonlocal fractional order differential equations with changing-sign singular perturbation," Applied Mathematical Modelling, vol. 39, no. 21, pp. 6543-6552, 2015.

[23] X. Zhang, L. Liu, and Y. Wu, "Multiple positive solutions of a singular fractional differential equation with negatively perturbed term," Mathematical and Computer Modelling, vol. 55, no. 3-4, pp. 1263-1274, 2012.

[24] C. Chen, X. Zhang, G. Zhang, and Y. Zhang, "A two-grid finite element method for nonlinear parabolic integro-differential equations," International Journal of Computer Mathematics, vol. 96, no. 10, pp. 2010-2023, 2019.

[25] D. Guo and V. Lakshmikantham, Nonlinear Problems in Abstract Cones, Academic Press, Orlando, FL, USA, 1988. 